\title{
Genome-wide analysis of salt-responsive and novel microRNAs in Populus euphratica by deep sequencing
}

\author{
Jingna Si, Tao Zhou, Wenhao Bo, Fang Xu, Rongling $\mathrm{Wu}^{*}$ \\ From International Symposium on Quantitative Genetics and Genomics of Woody Plants \\ Nantong, China. 16-18 August 2013
}

\begin{abstract}
Background: Populus euphratica is a representative model woody plant species for studying resistance to abiotic stresses such as drought and salt. Salt stress is one of the most common environmental factors that affect plant growth and development. MicroRNAs (miRNAs) are small, noncoding RNAs that have important regulatory functions in plant growth, development, and response to abiotic stress.

Results: To investigate the miRNAs involved in the salt-stress response, we constructed four small cDNA libraries from P. euphratica plantlets treated with or without salt (300 mM NaCl, 3 days) in either the root or leaf. Using highthroughput sequencing to identify miRNAs, we found 164 conserved miRNAs belonging to 44 families. Of these, 136 novel miRNAs were from the leaf, and 128 novel miRNAs were from the root. In response to salt stress, 95 miRNAs belonging to 46 conserved miRNAs families changed significantly, with 56 miRNAs upregulated and 39 miRNAs downregulated in the leaf. A comparison of the leaf and root tissues revealed 155 miRNAs belonging to 63 families with significantly altered expression, including 84 upregulated and 71 downregulated miRNAs. Furthermore, 479 target genes in the root and 541 targets of novel miRNAs in the leaf were predicted, and functional information was annotated using the Gene Ontology and Kyoto Encyclopedia of Genes and Genomes databases.
\end{abstract}

Conclusions: This study provides a novel visual field for understanding the regulatory roles of miRNAs in response to salt stress in Populus.

\section{Background}

MicroRNAs (miRNAs) are a class of endogenous noncoding single-stranded RNAs of about 21-23 nucleotides (nt) in length, which participate in the posttranscriptional regulation of flora and fauna gene expression [1,2]. The miRNAs were first discovered in Caenorhabditis elegans in 1993 [3]. To date, 24,521 miRNAs have been identified in animals [4], plants [4], and viruses [5] (MiRBase Release20: June 2013) [6]. Most miRNAs exist as single copies, multiple copies, or gene clusters in the genome. The identification and analysis of plant miRNAs have focused on several model species including Arabidopsis

\footnotetext{
* Correspondence: RWu@phs.psu.edu

Center for Computational Biology, National Engineering Laboratory for Tree Breeding, College of Biological Sciences and Biotechnology, Beijing Forestry
} University, Beijing 100083, China families have been identified in Populus euphratica in the miRBase Release20. Recent findings showed that miRNAs play important roles in response to various abiotic stresses in plants, including high salinity [7,8], drought [9-12], low temperatures $[7,13]$, oxidative stress [14], hypoxic stress [15,16], UV-B radiation [17], and mechanical stress $[17,18]$.

Salt stress is one of the major blocks in agricultural and forestry growth and production in modern times. To resist high-salinity stress and sustain their growth, plants have evolved multiple gene regulatory profiles to regulate water and ion balance and maintain normal photosynthesis. These regulatory genes are involved in a series of physiological, biochemical, and cellular processes essential for energy metabolism, photosynthesis, 
signal transduction, transcription, and protein biosynthesis and decay. In recent years, several studies have reported on the transcriptional regulation of specific miRNAs and genes in response to the salt-stress environment [19-21]. Using the microarray method, Liu et al. discovered 10 miRNAs in Arabidopsis that showed differential expression under salt-treatment conditions [7]. In addition, miR393 was strongly upregulated when treated with $300 \mathrm{mM} \mathrm{NaCl}$ [22]. In rice, miR169g was upregulated during high-salinity stress, and the transgenic plants that overexpressed miR393 were more sensitive to salt treatment than control plants [23,24]. In microarray studies focused on forestry species, several miRNAs such as miR395, miR398, and miR399 in Populus tremula were upregulated under salt stress. Notably, however, miR398 was downregulated in salt-treated Arabidopsis [25]. MiR168, miR1444, and miR1446 expression levels were greatly altered under salt conditions in P. euphratica [26]. In Populus trichocarpa, the expression of a large number of miRNAs was influenced by many environmental factors including salt stress [27]. Despite these advances, the regulatory mechanisms of miRNAs in plant growth and development remain undefined, and more in-depth studies on miRNA expression in response to salt stress in plants are required, especially for $P$. euphratica, a tree species known for its strong resistance to salinity. In addition, little research has focused on the systemic identification of saltresponsive miRNAs in $P$. euphratica at the genome level using high-throughput sequencing.

The poplar species $P$. euphratica grows almost exclusively in the desert. A great majority of $P$. euphratica are grown in China, and $90 \%$ of these are distributed in the Tarim River Basin in Xinjiang Province [28]. P. euphratica has a high tolerance for salinity, drought, cold, and wind, which makes it one of the only tree species in the Taklimakan Desert [29]. Thus, P. euphratica is widely accepted as an ideal model species for studying the abiotic stress resistance of woody plants [30]. Studies on P. euphratica miRNAs in response to salt stress may expand the understanding of the mechanism of gene function and regulation in resistance to stress [31]. In this study, the high-throughput sequencing method, which has been used widely for miRNA research [10-13,32,33], was used to identify conserved and novel miRNAs of $P$. euphratica in the roots and leaves. We analyzed the expression levels of these miRNAs in the different tissues under salt treatment and in controls, and investigated the potential roles of their target genes.

\section{Methods}

Plant materials and stress treatment

The experimental materials were poplar cutting clones from 2-year-old robust $P$. euphratica plantlets from Korla, Xinjiang Province. Briefly, seedlings were grown $10 \mathrm{~cm}$ apart above ground in a greenhouse for 6 months; thereafter, the seedlings were amputated and planted in $2-\mathrm{L}$ plastic pots. After the section buds reached $10-20 \mathrm{~cm}$, healthy sprouts were selected, cut to approximately $10 \mathrm{~cm}$ stem lengths, soaked in $0.01 \%$ ABT1 solution for $30 \mathrm{~min}$, and then inserted into a mixture of vermiculite, perlite, and peat in a 1:1:1 matrix to cultivate and maintain adequate soil moisture. Seedlings were grown in a greenhouse for 1 year, and 10 clones were selected for the experiments.

For the salt treatment, $10 \mathrm{P}$. euphratica plantlets were grown in 2-L plastic containers in a greenhouse at Beijing Forestry University. In the salt-treated group $(n=5)$ plants were watered using a $300 \mathrm{mM} \mathrm{NaCl}$ solution to saturate the soil two times per week. The control group $(n=5)$ was irrigated using pure water twice weekly. After 3 weeks, the leaves and roots from the salt-treated and control groups were selected, frozen immediately in liquid nitrogen, and stored at $-80^{\circ} \mathrm{C}$ until RNA extraction.

\section{RNA extraction followed by construction and sequencing of small RNA libraries}

Total RNA was extracted three times from the salt-treated and control plantlets using a modified cetyltrimethylammonium bromide (CTAB) procedure [34]. Four sRNA libraries were constructed $[11,35]$ using RNA extracted from the salt-treated leaves (3dSL), salt-treated roots (3dSR), untreated leaves (control, 3dCKL), and untreated roots (control, 3dCKR). After detecting the RNA integrity with $2 \%$ agarose gel electrophoresis, the four libraries were sequenced by Solexa sequencing (Illumina, USA) at the Beijing Genomics Institute (BGI), Shenzhen, China.

\section{Bioinformatic analysis of miRNAs and target prediction of miRNAs}

The original image data obtained from the Solexa sequencer became the raw reads in the base-calling procedure. Next, the contaminant reads were removed such as the sequences with and without insert fragments, lowquality reads, polyA tails, and sequences shorter than $18 \mathrm{nt}$ or longer than $30 \mathrm{nt}$. The remaining clean reads were used to analyze small RNA sequence types, sequence number, and sequence length distribution, then mapped to the Populus genome (http://www.phytozome. net/poplar) using SOAP [36]. The sequences with a perfect match were analyzed further. We excluded the noncoding RNAs identified as rRNAs, scRNAs, snoRNAs, snRNAs, and tRNAs that were annotated by comparisons with the NCBI GenBank (http://www.ncbi.nih.gov/GenBank/) [37] and Rfam10.1 (http://rfam.sanger.ac.uk/) databases. Small RNA tags corresponding to mRNA exons and introns in the Populus genome by Overlap were also excluded from further analysis. The remaining unannotated small RNAs were annotated by aligning to 
miRBase18.0 (http://www.mirbase.org/index.shtml) [38], allowing two mismatches at most. To identify the characteristic secondary structures, the conserved miRNAs weres screened using the program RNAfold (http://www. tbi.univie.ac.at/ ivo/RNA/ViennaRNA-1.8.1.tar.gz) [39]. The remaining reads were used to predict new miRNAs using the prediction software Mireap (http://sourceforge. net/projects/mireap). New criteria that considered the whole developmental progress of miRNAs were used for distinguishing candidate novel miRNAs [40]. The target genes of new miRNAs were predicted according to previous studies [41-43], allowing at most four mismatches in the miRNA/target gene duplex. The predicted target genes were used to annotate the functions and pathways using the Gene Ontology (GO, http://www.geneontology. org/) and Kyoto Encyclopedia of Genes and Genomes (KEGG, http://www.genome.jp/kegg/) [44] databases.

\section{Differential expression analysis of miRNAs under salt stress}

To investigate the expression difference of these samples, we modified the frequency of each miRNA in the different libraries to 1 million (normalized expression = miRNA expression count $* 1,000,000 /$ total number of clean reads). To reduce error, miRNAs were removed that showed normalized expression of less than 1 . The fold-change between the treatment and control samples was calculated as follows:

$$
\text { Fold }- \text { change }=\log _{2}^{\text {treatment } / \text { control }}
$$

The $P$-value represented the significant degree of miRNA expression differences between samples, with the smaller $P$-value signifying a greater significant difference. It was calculated as follows:

$$
\begin{aligned}
& p(x \mid y)=\left(\frac{N_{2}}{N_{1}}\right) \frac{(x+y) !}{x ! y !\left(1+\frac{N_{2}}{N_{1}}\right)^{(x+y+1)}} \\
& C\left(y \leq y_{\min } \mid x\right)=\sum_{y=0}^{\gamma \leq \gamma_{\min }} p(y \mid x) \\
& D\left(y \geq y_{\max } \mid x\right)=\sum_{y \geq y_{\max }}^{\propto} p(y \mid x)
\end{aligned}
$$

In the formulation, $\mathrm{N} 1$ and $\mathrm{N} 2$ mean the total number of sample1 and sample2. Meanwhile, $x$ and $y$ represent the expression level of known miRNAs respectively. The smaller $\mathrm{P}$ value stands for the greater difference of miRNAs expression between two samples, which was in accord with the statistical law.

\section{Results}

Analysis of small RNAs in salt-treated plants

To identify salt-responsive miRNAs in P. euphratica in leaves and roots, four sRNA libraries were constructed from $P$. euphratica plantlets treated with $\mathrm{NaCl}$ solutions or pure water. In total, 16.3 million raw reads were produced in the salt-treated and the control leaves (3dSL and $3 \mathrm{dCKL}$, respectively), 17.7 million raw reads were produced in salt-treated roots (3dSR), and 16.2 million reads were produced in control roots (3dCKR).

After removing redundant reads and those that had low quality, 3' adapter null, insert null, 5' adapter contaminants, and polyA tails, more than $95 \%$ of the reads were obtained as clean reads with lengths ranging from 18 to 30 nt. At least $70 \%$ of the reads had lengths of 20 $24 \mathrm{nt}$ (Figure 1). The most abundant reads were $21 \mathrm{nt}$ in length, followed by 24-nt reads (Figure 1), a finding that was consistent with previous results $[11,45,46]$. This phenomenon was observed not only in leaves, but also in roots. Notably, $49.31 \%$ of the reads were $21 \mathrm{nt}$ in $3 \mathrm{dSL}$, and $32.58 \%$ were $21 \mathrm{nt}$ in $3 \mathrm{dSR}$, suggesting that a significantly greater number of 21-nt reads in leaves participated in the salt-response process. In addition, the number of 21-nt reads in salt-treated libraries was slightly more than those from the control libraries,

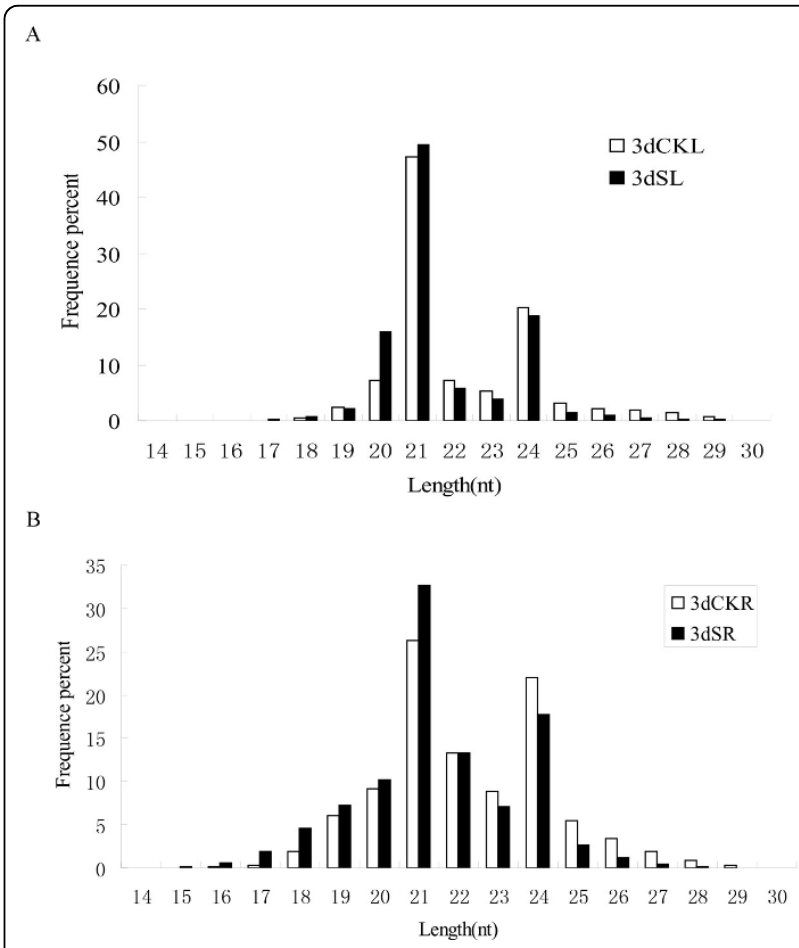

Figure 1 Size distribution of small RNA reads in different libraries. (A) Control (3dCKL) and salt stress (3dSL) from Populus euphratica leaf libraries. (B) Control (3dCKR) and salt stress (3dSR) from $P$. euphratica root libraries. 
suggesting that more 21 -nt reads were induced in response to salt stress. Furthermore, greater numbers of 24-nt reads were produced under salt conditions.

The sRNAs were mapped to the Populus genome (http:// www.phytozome.net/poplar), miRBase 20.0 version (http:// microrna.sanger.ac.uk/sequences), GenBank (http://www. ncbi.nih.gov/genbank/), and Rfam (http://rfam.sanger.ac. $\mathrm{uk} /$ ) databases. The sequenced sRNAs were annotated and classified into six categories: exons (sense, antisense), introns (sense, antisense), known miRNAs (identified miRNAs in miRBase 20.0), rRNAs (i.e., rRNA, tRNA, snRNA, scRNA, siRNA, and snoRNA) repeat-associated RNAs, and unknown sRNAs (Figure 2). The results showed that the proportion of known miRNAs increased from $37.24 \%$ to $40.30 \%$ in response to salt stress in leaves and increased from $12.57 \%$ to $18.70 \%$ under salt stress in roots (Figure 2). This result implies that miRNAs in leaves are more abundant than those in roots, and that these miRNAs had an important function under salt stress. However, the percentage of unknown sRNAs reads increased slightly from $28.36 \%$ (3dCKL) to $30.11 \%$ (3dSL) and decreased from $54.42 \%$ (3dCKR) to $50.67 \%$ (3dSR), indicating that large numbers of unknown salt-responsive sRNAs were unidentified (Figure 2). The repeats, exons, and introns revealed little change under the different conditions.

The common and specific sequences were analyzed in different libraries. In total, 3,923,163 unique sRNAs and $32,010,578$ total sRNAs were identified in the two leaf libraries (Table 1). Although $13.68 \%$ of the sequences were coincident in the two libraries, these sRNAs covered $87.19 \%$ of the total sRNAs. In comparison, $6,861,893$ unique sRNAs and 32,900,213 total sRNAs were found in the two roots libraries (Table 2). Although $12.40 \%$ of the sequences were coincident in both root libraries, these sRNAs covered $77.72 \%$ of all sRNAs. The specific percentage in both the salt-treated libraries for unique sRNA reads was about $3 \%$ higher than that in the control libraries.

\section{Identification of conserved miRNAs in $P$. euphratica}

To identify conserved miRNAs in $P$. euphratica, sequenced reads were searched by alignment against the known miRNA database in miRBase 20.0, allowing two mismatches; $10,202,10,672,10,471$, and 11,037 sequences

A

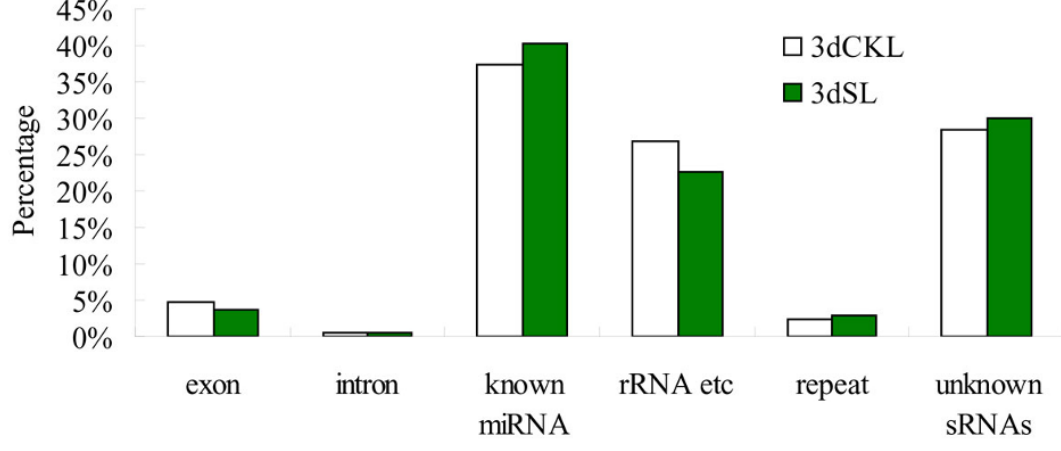

B

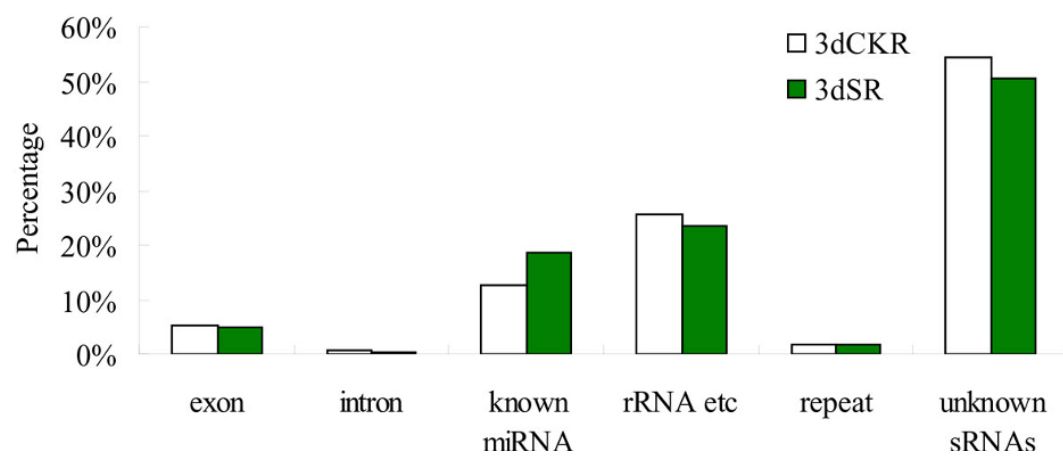

Figure 2 Distribution of different sRNA annotation categories in total reads of control and salt-stress libraries. (A) Control (3dCKL) and salt stress (3dSL) from Populus euphratica leaf libraries. (B) Control (3dCKR) and salt stress (3dSR) from P. euphratica root libraries. 
Table 1 Summary of common and specific sequences between 3dSL and 3dCKL libraries

\begin{tabular}{ccccc}
\hline Class & Unique sRNA & Percentage & Total sRNA & Percentage \\
\hline Total sRNAs & 3923163 & 100.00 & 32010578 & 100.00 \\
3dSL \& 3dCKL & 536735 & 13.68 & 27911344 & 87.19 \\
3dSL-specific & 1754216 & 44.71 & 2092797 & 6.54 \\
3dCKL-specific & 1632212 & 41.60 & 2006437 & 6.27 \\
\hline
\end{tabular}

Table 2 Summary of common and specific sequences between 3dSR and 3dCKR libraries

\begin{tabular}{ccccc}
\hline Class & Unique sRNA & Percentage & Total sRNA & Percentage \\
\hline Total sRNAs & 6861893 & 100.00 & 32900213 & 100.00 \\
3dCKR \& 3dSR & 850624 & 12.40 & 25568498 & 77.72 \\
3dCKR-specific & 2903793 & 42.32 & 3534735 & 10.74 \\
3dSR-specific & 3107476 & 45.29 & 3796980 & 11.54 \\
\hline
\end{tabular}

were identified in the $3 \mathrm{dSL}, 3 \mathrm{dSR}, 3 \mathrm{dCKL}$, and $3 \mathrm{dCKR}$ libraries, respectively. The sequences with low expression levels ( $<5$ reads) were removed, and the remaining reads were screened to find the characteristic hairpin structures in the Populus genome. Finally, we obtained 164 conserved miRNAs belonging to 44 families. Moreover,
59 miRNA* sequences were identified; these complementary sequence were considered direct evidence for the actual miRNAs [40]. In both the leaf and root libraries, the family of peu-miR156 was the most abundant, comprising about $60-70 \%$ of the total conserved miRNA reads. Moreover, the amount of peu-miR156 in leaves remained almost the same regardless of salt- or control-treatment; however, peu-miR156 in roots increased from $57.4 \%$ to $72.4 \%$ under the salt treatment (Figure 3). This result shows that a greater number of peu-miR156 members respond to salt stress in roots than in leaves. Peu-miR166 and peu-miR167 are the second-most enriched miRNA family (approximately 10\% in these libraries) (Figure 3) and showed no obvious changes in salt stress. Meanwhile, some miRNA families were moderately represented in P. euphratica, such as peu-miR167, peu-miR169, peumiR172, peu-miR827, peu-miR2119, and peu-miR5020. Among the conserved miRNAs families, the expressions of peu-miR393, peu-miR645, peu-miR860, and peumiR1444 decreased significantly under salt stress in roots, but not in leaves. Peu-miR408 significantly increased in leaves (fold-change $=3.3$ ) and decreased in roots (fold-change $=-3.60$ ). For peu-miR160 and peumiR391, tenfold more reads were identified in $3 \mathrm{dSL}$

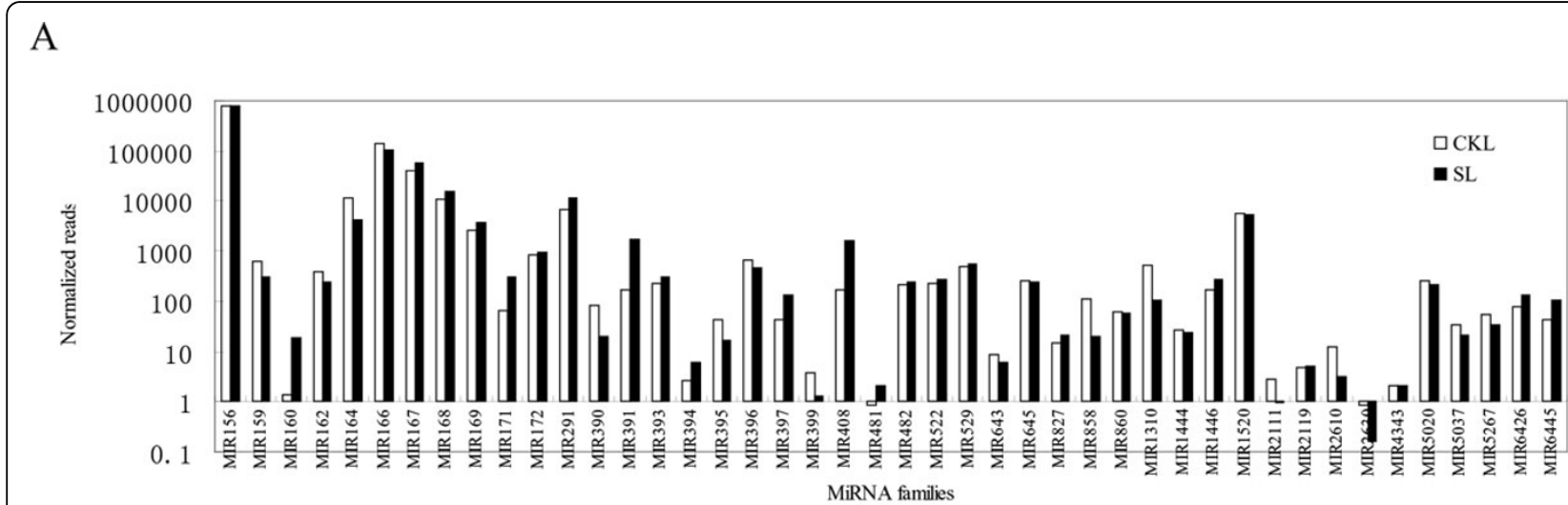

B

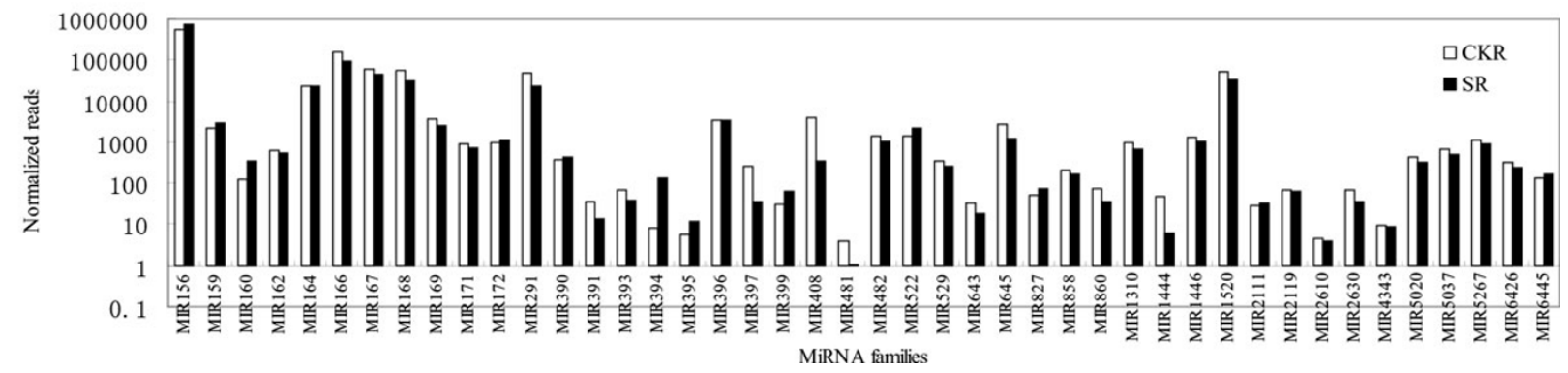

Figure 3 Differential expression of conserved miRNA families in Populus euphratica. (A) Libraries constructed from salt-treated and control leaf tissue. (B) Libraries constructed from salt-treated and control root tissue. 
than in 3dCKL, and peu-miR394 was identified 17 times more in 3dSR than in 3dCKR.

\section{Identification of novel miRNAs in $P$. euphratica}

According to the new annotation criteria of novel miRNAs [40], the detection of miRNA"s is robust proof for the existence of their miRNAs. In this study, we found 136 miRNA sequences in the leaf and 128 miRNAs in the root (Additional files 1 and 2). These sequences have hairpin structures and miRNA*s. The average precursor length was $160 \mathrm{nt}$ in the leaf and $151 \mathrm{nt}$ in the root. The minimal folding free energy varied from -198.7 to -19.1 $\mathrm{kcal} / \mathrm{mol}$, with an average of $-54.36 \mathrm{kcal} / \mathrm{mol}$ in the leaf. In comparison, the minimal folding free energy varied from -136.2 to $-19.42 \mathrm{kcal} / \mathrm{mol}$, with an average of -53.01 $\mathrm{kcal} / \mathrm{mol}$ in the root. In both the leaf and root, most new miRNAs were $21 \mathrm{nt}$ long and had guanine (G) as their first nucleotide. More than half of the new miRNAs were located in the $5^{\prime}$ arm of the stem-loop. The novel miRNAs had different expression levels; some had high expression levels, such as Peu-sM31 in leaves and PeusM49 in roots, with normalized reads close to $1,000,000$. To identify possible homologs with existing miRNAs, we searched these novel miRNAs against plant miRNAs in the miRBase database, and found 20 homologs in P. trichocarpa and 15 homologs in A. thaliana and Glycine max. Several novel miRNAs were identified in only one of the libraries, probably because the sequencing depth provided insufficient coverage of all the miRNAs; alternatively, the expression of some miRNAs might have been turned on or turned off suddenly by salt stress.

\section{Identification of salt-responsive miRNAs}

To identify the salt-responsive miRNAs among the deep sequencing reads, we performed differential expression analysis of miRNAs between two libraries. miRNAs with very low expression levels (normalized reads $<1$ ) were removed. miRNAs that showed a fold-change greater than 0.5 or less than -0.5 with $P$-values less than 0.05 were considered to be downregulated or upregulated, respectively. There are totally 157 miRNAs detected from 3dSL and 3d CKL libraries. Between the 3dSL and 3dCKL libraries, we identified 95 differentially regulated miRNAs belonging to 46 miRNAs families, including 39 downregulated and 56 upregulated miRNAs in response to salt stress (Additional file 3 and Figure 4A). The greatest change between the two libraries was in miR6424, which showed a change greater than fivefold. In addition, expression differences between the 3dSL and 3dSR libraries revealed $155 \mathrm{miR}$ NAs from the totally 188 miRNAs belonging to 63 families, of which 84 were upregulated and 71 were downregulated from leaf to root tissue (Additional file 4 and Figure 4B). These significant changes in miRNAs expression between different libraries revealed that miRNAs are sensitive and responsive to salt stress; furthermore, a greater number of differentially expressed miRNAs play important roles in Populus root than leaf tissues.

\section{Prediction of target genes of novel miRNAs in $P$. euphratica}

The target gene predictions were carried out as described previously $[41,42]$ with default parameters. The functions of target genes were annotated using the GO and KEGG [44]. In root samples, 479 targets were identified for the novel miRNAs, with an average of six targets per miRNA (range: 1-43; Additional file 5). In comparison, 541 targets for novel miRNAs were identified in leaves, with an average of six targets per miRNA (range: 1-67; Additional file 6). The GO and KEGG databases predicted a wide distribution of target functions such as plant development, cell apoptosis, hormone regulation, cell defense, disease resistance, and the electron transfer chain. For example, according to the GO database, the most frequent gene targets were related to protein tyrosine phosphatase activity (GO term: 0004725). In contrast, the KEGG database inferred that most targets focused on various pathways including those involved in plantpathogen interactions, the cell cycle, cytosolic DNA-sensing pathway, RNA polymerase, and Huntington's disease. The diverse functions of these target participants revealed that the novel miRNAs and their target genes play important roles in the developmental and regulatory processes in P. euphratica.

\section{Discussion}

More than 954 million hectares of saline land areas exist worldwide, with about $10 \%$ of them distributed in China. Salinity stress has become a serious threat to plant growth and development that can not be ignored [47]. Many miRNAs and genes involved in the high-salinity stress response in plants have been identified $[19,48]$; however, little research has focused on the saltresponsive miRNAs in woody plants on a genome-wide scale. In this study, we constructed four small RNA cDNA libraries from the root or leaf tissues in $P$. euphratica plantlets treated with or without salt $(300$ $\mathrm{mM} \mathrm{NaCl}, 3$ days). By high-throughput sequencing, we identified 164 conserved miRNAs belonging to 44 families (136 novel miRNAs in leaf and 128 novel miRNAs in root), and provided a genome-wide expression profile of miRNAs in response to salt-stress conditions. Furthermore, we identified 479 target genes in root and 541 targets in leaf of novel miRNAs and annotated them by mapping to the GO and KEGG databases. These results strengthen the understanding of the roles of salt-responsive miRNAs and may provide an important reference for improving Populus resistance to salt stress. 


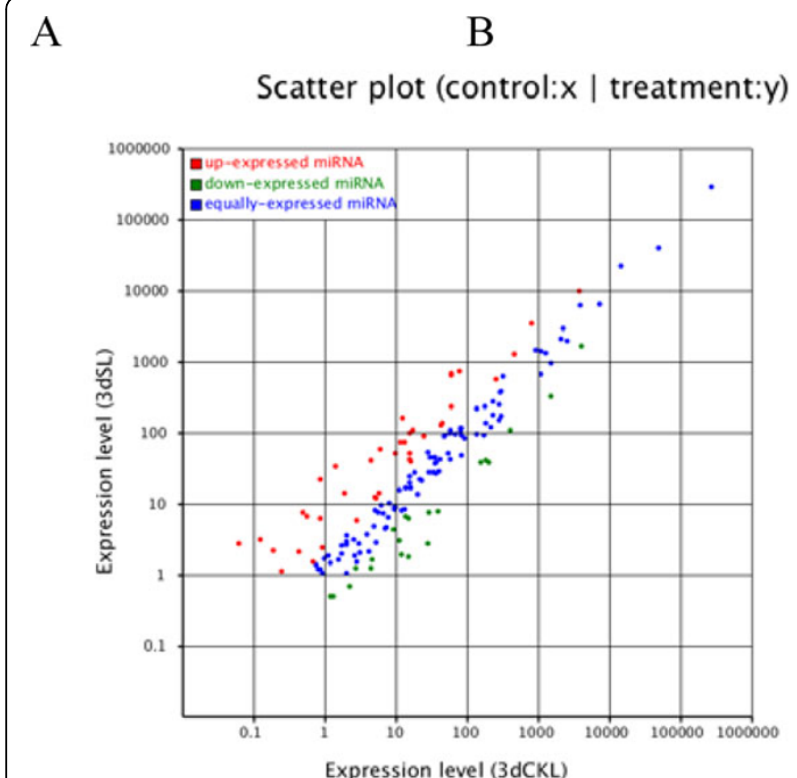

C

D

Scatter plot (control:x | treatment:y)

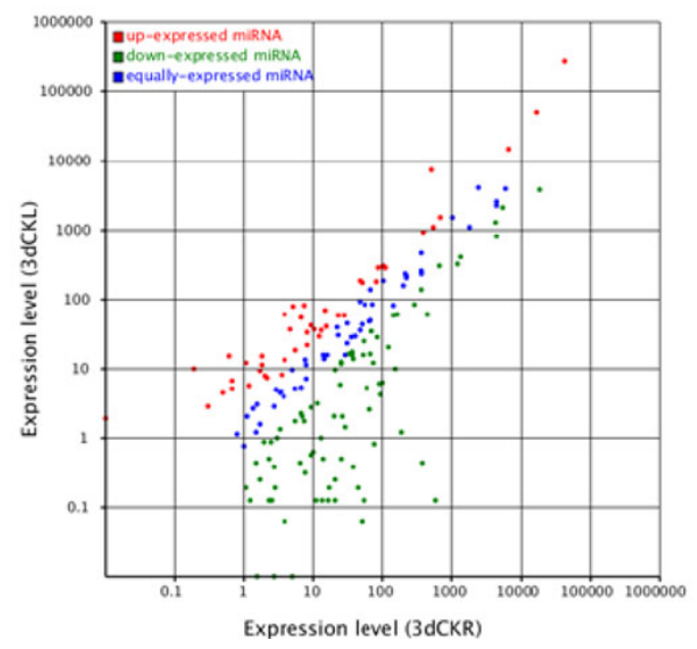

Scatter plot (control:x | treatment:y)

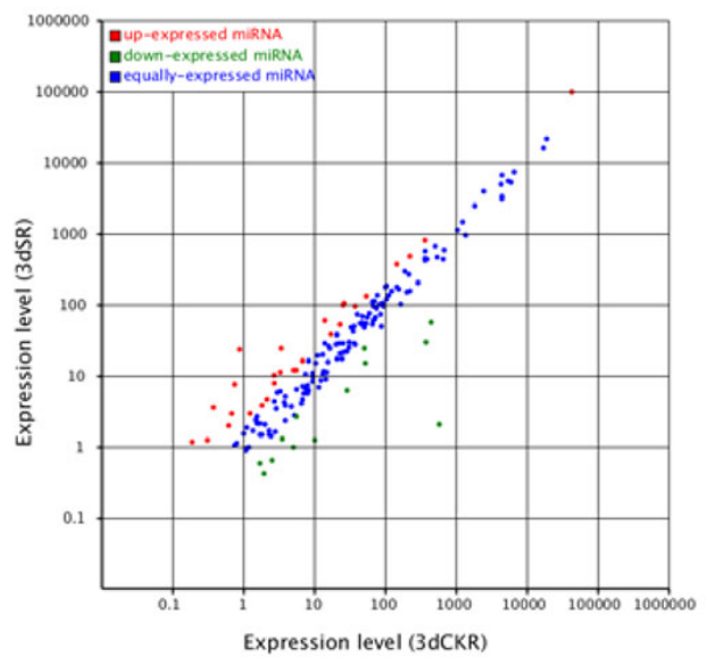

Scatter plot (control:x | treatment:y)

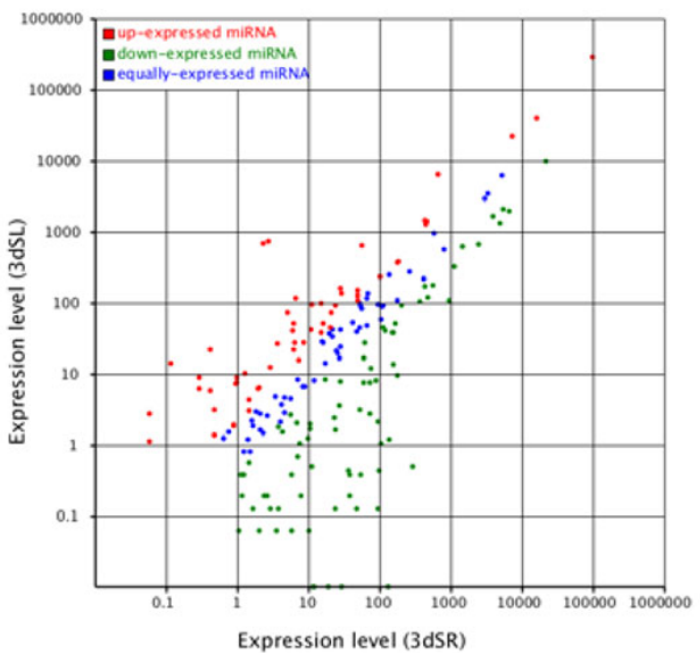

Figure 4 Expression differences in conserved miRNAs between libraries constructed from salt-treated and untreated tissues. (A) The salt-treated leaf (3dSL) and control leaf (3dCKL) libraries. (B) The salt-treated root (3dSR) and control root (3dSR) libraries. (C) The control root $(3 \mathrm{dCKR})$ and control leaf (3dCKL) libraries. (D) The salt-treated leaf (3dSL) and salt-treated root (3dSR) libraries. Each point in the figure represents a miRNA; the $x$ - and $y$-axes represent the miRNA expression levels in the two samples; red represents miRNAs with ratios $>2$; blue represents miRNAs with ratios $\geq 1 / 2$ and $\leq 2$; green represents miRNAs with ratios $<1 / 2$; ratios are the normalized expression in the salt-treated sample: normalized expression in the control sample.

In response to salt stress, 95 miRNAs belonging to 46 conserved miRNAs families were identified, containing 56 upregulated miRNAs and 39 downregulated miRNAs in the leaf. In a comparison of leaf and root tissues, we identified 155 miRNAs belonging to 63 families with significantly altered expression, including 84 upregulated and 71 downregulated miRNAs. An analysis of the changed expression of conserved miRNAs showed 21 miRNAs in the leaf (Additional file 3 and Figure 4A) and 14 miRNAs (Additional file 4 and Figure 4B) in the root that were significantly downregulated in response to salt stress. At the same time, 39 upregulated miRNAs 
were found in the leaf (Additional file 3 and Figure 4A) and 28 upregulated miRNAs (Additional file 4 and Figure 4B) were found in root tissues. In contrast, 60 miRNAs were downregulated and 54 were upregulated (Additional file 7 and Figure 4C) in the 3dCKR sample compared to the $3 \mathrm{dCKL}$ sample; furthermore, 77 were downregulated and 56 were upregulated (Additional file 8 and Figure 4D) in the 3dSR sample compared to the 3dSL sample. The expression of several miRNAs changed in response to salt stress; however, a greater difference in miRNA expression was detected when the root and leaf tissues were compared. A similar phenomenon was observed in the differential expression of the novel miRNAs: 16 upregulated and 38 downregulated predicted miRNAs were identified between the $3 \mathrm{dCKL}$ and $3 \mathrm{dSL}$ samples (Additional file 9 and Figure 5A), and 18
A

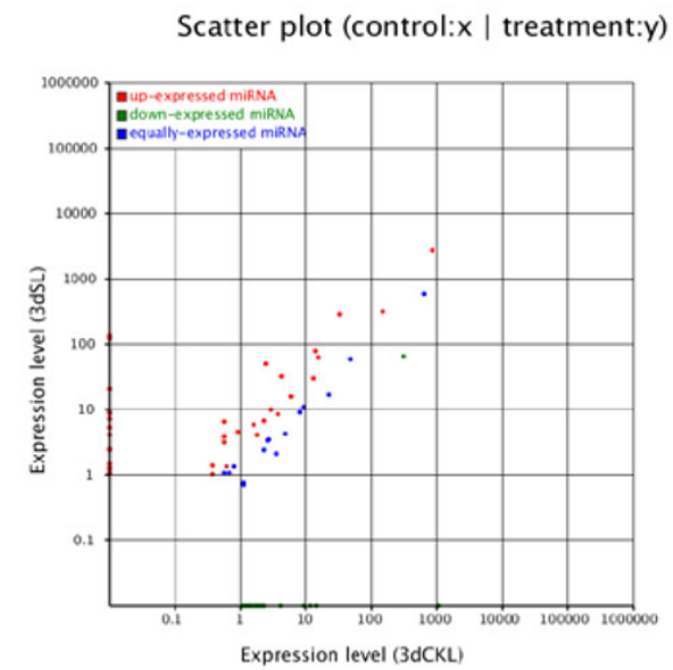

C

Scatter plot (control:x | treatment:y)

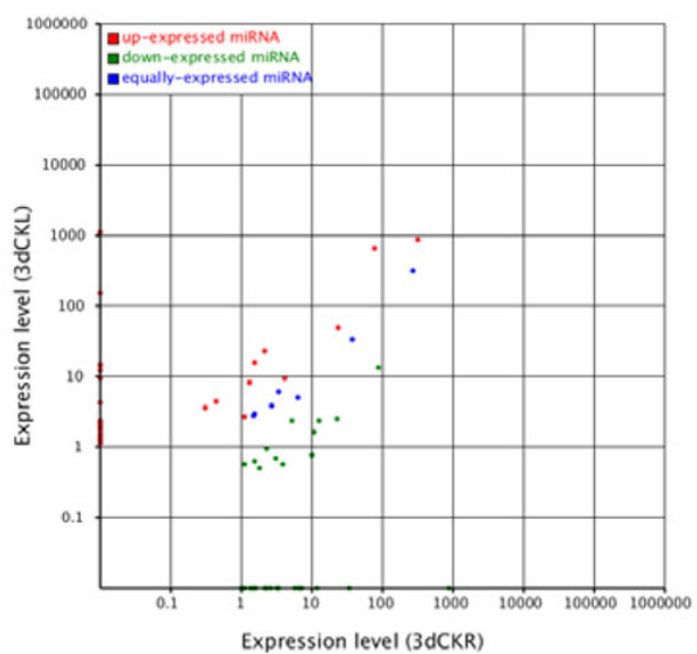

B

Scatter plot (control:x | treatment:y)

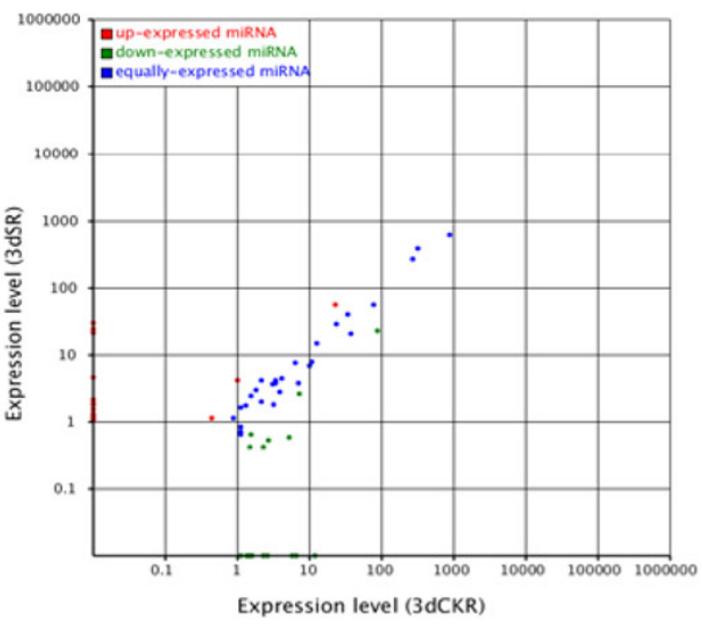

D

Scatter plot (control: $x \mid$ treatment:y)

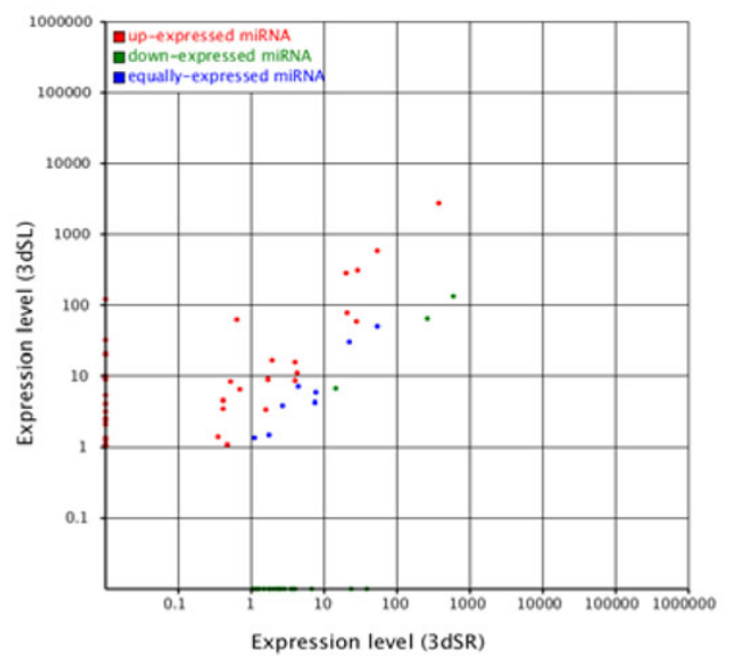

Figure 5 Expression differences of novel miRNAs between libraries constructed from salt-treated and untreated tissues. (A) The salt-treated leaf (3dSL) and control leaf (3dCKL) libraries. (B) The salt-treated root (3dSR) and control root (3dSR) libraries. (C) The control root (3dCKR) and control leaf (3dCKL) libraries. (D) The salt-treated leaf (3dSL) and salt-treated root (3dSR) libraries. Each point in the figure represents a miRNA; the $x$ - and $y$-axes represent the miRNA expression levels in the two samples; red represents miRNAs with ratios $>2$; blue represents miRNAs with ratios $\geq 1 / 2$ and $\leq 2$; green represents miRNAs with ratios $<1 / 2$; ratios are the normalized expression in the salt-treated sample:normalized expression in the control sample. 
upregulated and 16 downregulated novel miRNAs were identified between the 3dCKR and 3dSR samples (Additional file 10 and Figure 5B). In contrast, 32 upregulated and 30 downregulated predicted miRNAs were found between the $3 \mathrm{dCKL}$ and $3 \mathrm{dCKR}$ samples (Additional file 11 and Figure 5C), and 23 upregulated and 41 downregulated novel miRNAs were found between the 3dSR and 3dSL samples (Additional file 12 and Figure 5D). The phenomenon suggested that the miRNA expression changes occurred not only in response to abiotic stress, but also in different tissues.

Peu-miR394a, which targets the gene encoding F-box proteins, was significantly upregulated in response to salt stress both in the leaf and root tissue; the same tendency was observed in ath-miR394 under high-salinity stress in Arabidopsis $[7,22]$. Another related miRNA, peu-miR393a, was upregulated in the leaf, but with weak expression changes in the root. The diverse expression trends observed for these two miRNAs in different species may indicate that F-box proteins play varied roles under saltstress conditions in different regulated pathways [49-51]. Figure 3 shows that peu-miR160 expression was significantly upregulated in both tissues, in contrast to the findings in Populus tomentosa. MiR160 target genes encoded B3 DNA-binding domain proteins and auxin response factor (ARF). ARF affects various facets of plant growth and development and response to environmental changes [52-54]. Plant auxins act as signals for cell division, elongation, or differentiation and play important roles in lateral root formation, apical dominance, and tropisms [55]. In Vigna, the expression of vun-miR160a was clearly upregulated under salt conditions [56]; however, in maize, the expression of miR160a and miR160b was induced by $5 \mathrm{~h}$ of salt treatment but reduced by $24 \mathrm{~h}$ of salt treatment. The differentially altered expression in different species indicated that peu-miR160 might play a complex role in salt-stress resistance by affecting the auxin signaling pathways.

MiR168, miR169, and miR1444 were downregulated by salt shock in the root tissues. However, miR168 and miR169 were induced by salt in the leaf tissues, which was described in a previous study [26]. The induction of miR169 by salt stress was also reported in rice [23]. In P. tomentosa, the expression trends of miR168, miR169, and miR1444 were all restrained by salt stress [57]. MiR168 controls the Argonaute 1 (AGO1) gene and acts as a miRNA pathway regulator [27]. The altered expression of miR168 in salt-treated plants suggests that $A G O 1$ may play important roles in response to salt stress. The CCAAT-binding transcription factors in Arabidopsis [58] and rice [23] are encoded by the target gene of miR169, which has been reported to play an important regulatory role in the response to salt stress. Polyphenol oxidase (ppo) genes, the targets of miR1444a, were found to be involved in the resistance of abiotic stress in plants. Studies have reported that Ptc-miR1444 might be involved in stress resistance in P. trichocarpa through the cleavage of ppo genes and disease resistance protein genes [27].

Peu-miR398 was significantly upregulated in the leaf under salt stress (Additional file 3) and downregulated in the root in response to salt. The expression level of miR398b was restrained after 9-12 h of salt treatment in Populus cathayana, whereas increased miR398 expression was observed in P. tremula [8]. The target gene of miR398b encodes copper/zinc superoxide dismutase (SODC), which showed an opposite expression pattern to miR398b [59]. Peu-miR395 expression decreased during salt stress, whereas miR395 was continuously induced under salt stress in P. tremula and maize [48]. MiR395 target genes encode adenosine phosphosulfate (APS) and Kelch motif proteins. APS and pyrophosphate anion $\left(\mathrm{P}_{2} \mathrm{O}_{7}{ }^{4-}\right)$ form ATP [60]. MiR396 greatly impacts plant leaf growth and development by repressing growth-regulating factor (GRF) transcription factors [61]. The zma-miR396 family, which targets genes encoding cytochrome oxidase subunit I, were found to be downregulated under salt conditions in maize [48]. In $P$. cathayana, miR396f was downregulated under salt stress, and the target genes encoding GRF were induced [59]. MiR396 in Arabidopsis was significantly upregulated in response to abiotic stresses including salt treatment [7]. The expression of the newly found peu-miR396b was upregulated in response to salt stress in the leaf with almost no expression changes in the root. This result is in accord with previous research [61]. Peu-miR396b was predicted to target several genes including those encoding protein tyrosine kinases, zinc finger proteins, and various other protein kinases. In rice and Arabidopsis, miR396c was induced by salt stress [62]. These findings suggest that the upregulation of miR396 increased the expression of its targeted genes to help plants adapt to saline environments.

In the identification of salt-responsive miRNAs, miRNAs with differential expression were identified with a nominal threshold of p-value $<0.05$ and fold_change $>1$ or $<-1$. No multiple testing adjustments were applied, such as FDR. The False Discovery Rate (FDR) of a set of predictions is the expected percent of false ones in the set of predictions. If the algorithm returns 100 genes with a false discovery rate of 0.3 then we should expect 70 of them to be correct. In the work, because of the small size of the libraries, we only used fold_change to filter the false positives with pvalue $<0.05$. In the future work, we should increase the sample size, and use popular multiple testing adjustments for reasons of credibility.

\section{Conclusions}

We constructed four small RNA cDNAs libraries from the root or leaf of salt-treated and pure water-treated P. euphratica plantlets. Genome-wide high-throughput 
sequencing was employed to identify and analyze saltinduced miRNAs in woody plants. We identified most of the known miRNAs in Populus and several conserved miRNAs not found previously in other Populus species $[45,63]$. Several novel miRNAs and miRNAs* were identified, and the presence of Dicer-like (DCL)-processed precursors was revealed, which are characteristics of bona fide miRNAs [40]. Furthermore, the targets of the novel miRNAs were predicted and functionally annotated. The predicted genes are involved in a broad range of functions in response to salt stress including signal transduction, transcriptional regulation, and energy metabolism. Through high-throughput sequencing, these findings provide solid evidence that miRNAs exist in Populus; moreover, they are distributed widely and differentially expressed under different salt conditions and different tissues. miRNAs in the root are more sensitive than those in the leaf in response to salt stress. The discovery and characterization of these miRNAs will help uncover the molecular mechanisms of abiotic stress resistance and elucidate new members of these pathways in Populus.

\section{Additional material}

Additional file 1: Novel microRNAs (miRNAs) identified in libraries constructed from the leaves of Populus euphratica that were treated with (3dSL) or without (control, $3 \mathrm{dCKL}$ ) salt.

Additional file 2: Novel miRNAs identified in libraries constructed from the roots of Populus euphratica that were treated with (3dSR) or without (control, 3dCKR) salt.

Additional file 3: Significant expression changes in conserved Populus euphratica miRNAs between libraries that were constructed from the leaves of salt-treated (3dSL) or control-treated (3dCKL) plants.

Additional file 4: Significant expression changes in conserved Populus euphratica miRNAs between libraries that were constructed from the roots of salt-treated (3dSR) and control-treated (3dCKR) plants.

Additional file 5: Summary of target genes of novel miRNAs from the root tissue.

Additional file 6: Summary of target genes of novel miRNAs from the leaf tissue.

Additional file 7: Significant expression changes in conserved Populus euphratica miRNAs between the control leaf (3dCKL) and control root (3dCKR) libraries.

Additional file 8: Significant expression changes in conserved miRNAs from salt-treated Populus euphratica in the leaf (3dSL) and root (3dSR) libraries.

Additional file 9: Significant expression changes in novel miRNAs in the leaves of salt-treated Populus euphratica (3dSL) and control-treated (3dCKL) libraries.

Additional file 10: Significant expression changes in novel miRNAs in the roots of salt-treated Populus euphratica (3dSR) and control-treated (3dCKR) libraries.

Additional file 11: Significant expression changes in novel miRNAs in control-treated Populus euphratica leaf (3dCKL) and root (3dCKR) libraries.

Additional file 12: Significant expression changes in novel miRNAs in the leaves of salt-treated Populus euphratica (3dSL) and untreated root (3dSR) libraries.

\section{Competing interests}

The corresponding author declares that there are no competing interests.

\section{Authors' contributions}

RLW designed the study, TZ prepared the CDNA libraries for miRNA sequencing, JNS analyzed the data and performed the bioinformatic analyses, JNS and RLW drafted the manuscript, and all authors contributed to editing the final version. All authors have read and approved the final manuscript.

\section{Funding}

Publication costs for this article came from Fundamental Research Funds for the Central Universities (TD2012-04), the Beijing Forestry University Young Scientist Fund (No. BLX2011007), the Research Fund for the Doctoral Program of Higher Education of China (20120014120011), Special Fund for Forest Scientific Research in the Public Welfare (201404102), NSF/IOS0923975, Changjiang Scholars Award and "Thousand-person Plan" Award.

\section{Declarations}

This article has been published as part of BMC Genetics Volume 15 Supplement 1, 2014: Selected articles from the International Symposium on Quantitative Genetics and Genomics of Woody Plants. The full contents of the supplement are available online at http://www.biomedcentral.com/ bmcgenet/supplements/15/S1.

Published: 20 June 2014

\section{References}

1. Bartel DP: MicroRNAs: genomics, biogenesis, mechanism, and function. Cell 2004, 116(2):281-97.

2. Jones-Rhoades MW, Bartel DP, Bartel B: MicroRNAS and their regulatory roles in plants. Annu Rev Plant Biol 2006, 57:19-53.

3. Lee RC, Feinbaum RL, Ambros V: The C. elegans heterochronic gene lin-4 encodes small RNAs with antisense complementarity to lin-14. Cell 1993, 75(5):843-54.

4. Zhang $B$, et al: Plant microRNA: a small regulatory molecule with big impact. Dev Biol 2006, 289(1):3-16.

5. Cullen BR: Viruses and microRNAs: RISCy interactions with serious consequences. Genes Dev 2011, 25(18):1881-94.

6. Griffiths-Jones $S$, et al: miRBase: microRNA sequences, targets and gene nomenclature. Nucleic Acids Res 2006, 34(Database):D140-4.

7. Liu HH, et al: Microarray-based analysis of stress-regulated microRNAs in Arabidopsis thaliana. Rna 2008, 14(5):836-43.

8. Jia $X$, et al: Differential and dynamic regulation of miR398 in response to $\mathrm{ABA}$ and salt stress in Populus tremula and Arabidopsis thaliana. Plant Mol Biol 2009, 71(1-2):51-9.

9. Trindade I, et al: miR398 and miR408 are up-regulated in response to water deficit in Medicago truncatula. Planta 2010, 231(3):705-16.

10. Zhou $L$, et al: Genome-wide identification and analysis of droughtresponsive microRNAs in Oryza sativa. J Exp Bot 2010, 61(15):4157-68.

11. Li B, et al: Genome-wide characterization of new and drought stress responsive microRNAs in Populus euphratica. J Exp Bot 2011, 62(11):3765-79.

12. Wang $T$, et al: Identification of drought-responsive microRNAs in Medicago truncatula by genome-wide high-throughput sequencing. BMC Genomics 2011, 12:367.

13. Zhang J, et al: Deep sequencing of Brachypodium small RNAs at the global genome level identifies microRNAs involved in cold stress response. BMC Genomics 2009, 10:449.

14. Sunkar R, Kapoor A, Zhu JK: Posttranscriptional induction of two $\mathrm{Cu} / \mathrm{Zn}$ superoxide dismutase genes in Arabidopsis is mediated by downregulation of miR398 and important for oxidative stress tolerance. Plant Cell 2006, 18(8):2051-65.

15. Licausi $F$, et al: Hypoxia responsive gene expression is mediated by various subsets of transcription factors and miRNAs that are determined by the actual oxygen availability. New Phytol 2011, 190(2):442-56

16. Zhang $Z$, et al: Submergence-responsive MicroRNAs are potentially involved in the regulation of morphological and metabolic adaptations in maize root cells. Ann Bot 2008, 102(4):509-19. 
17. Jia $X$, et al: UV-B-responsive microRNAs in Populus tremula. J Plant Physiol 2009, 166(18):2046-57.

18. Lu S, et al: Novel and mechanical stress-responsive MicroRNAs in Populus trichocarpa that are absent from Arabidopsis. Plant Cell 2005, 17(8):2186-203.

19. Seki $M$, et al: Monitoring the expression profiles of 7000 Arabidopsis genes under drought, cold and high-salinity stresses using a full-length cDNA microarray. Plant J 2002, 31(3):279-92.

20. Hirayama T, Shinozaki K: Research on plant abiotic stress responses in the post-genome era: past, present and future. Plant J 2010, 61(6):1041-52.

21. Vinocur $B$, Altman $A$ : Recent advances in engineering plant tolerance to abiotic stress: achievements and limitations. Curr Opin Biotechnol 2005, 16(2):123-32.

22. Sunkar R, Zhu JK: Novel and stress-regulated microRNAs and other small RNAs from Arabidopsis. Plant Cell 2004, 16(8):2001-19.

23. Zhao $B$, et al: Members of miR-169 family are induced by high salinity and transiently inhibit the NF-YA transcription factor. BMC Mol Biol 2009, 10:29.

24. Gao P, et al: osa-MIR393: a salinity- and alkaline stress-related microRNA gene. Mol Biol Rep 2011, 38(1):237-42

25. Jagadeeswaran G, Saini A, Sunkar R: Biotic and abiotic stress downregulate miR398 expression in Arabidopsis. Planta 2009, 229(4):1009-14.

26. Qin $Y$, et al: Expression profiles of precursor and mature microRNAs under dehydration and high salinity shock in Populus euphratica. Plant Cell Rep 2011, 30(10):1893-907.

27. Lu S, Sun YH, Chiang VL: Stress-responsive microRNAs in Populus. Plant J 2008, 55(1):131-51

28. Jian-Rong L, et al: Components of the heartwood of Populus euphratica from an ancient tomb. Nat compd 2008, 44(1):6-9.

29. Sharma DB, Singh B, Kumar K: Introduction of Populus euphratica in Indian semi-arid trans Gangetic plains. Ann For 1999, 7:1-9.

30. Ottow EA, et al: Molecular characterization of PeNhaD1: the first member of the $\mathrm{NhaD} \mathrm{Na+/H+}$ antiporter family of plant origin. Plant Mol Biol 2005, 58(1):75-88.

31. Chen J, Xia X, Yin W: Expression profiling and functional characterization of a DREB2-type gene from Populus euphratica. Biochem Biophys Res Commun 2009, 378(3):483-7.

32. Szittya $G$, et al: High-throughput sequencing of Medicago truncatula short RNAs identifies eight new miRNA families. BMC Genomics 2008, 9:593.

33. Zhao CZ, et al: Deep sequencing identifies novel and conserved microRNAs in peanuts (Arachis hypogaea L.). BMC Plant Biol 2010, 10:3.

34. Chang S, Puryear J, Cairney J: A simple and efficient method for isolating RNA from pine trees. Plant Mol Biol Rep 1993, 11:113-116.

35. Hafner $M$, et al: Identification of microRNAs and other small regulatory RNAs using CDNA library sequencing. Methods 2008, 44(1):3-12.

36. Li R, et al: SOAP: short oligonucleotide alignment program. Bioinformatics 2008, 24(5):713-4.

37. Benson DA, et al: GenBank. Nucleic Acids Res 2011, 40(Database):D48-53.

38. Griffiths-Jones $S$, et al: miRBase: tools for microRNA genomics. Nucleic Acids Res 2008, 36(Database):D154-8.

39. Mathews DH, et al: Expanded sequence dependence of thermodynamic parameters improves prediction of RNA secondary structure. $J$ Mol Biol 1999, 288(5):911-40.

40. Meyers BC, et al: Criteria for annotation of plant MicroRNAs. Plant Cell 2008, 20(12):3186-90

41. Allen $E$, et al: microRNA-directed phasing during trans-acting siRNA biogenesis in plants. Cell 2005, 121(2):207-21.

42. Schwab R, et al: Specific effects of microRNAs on the plant transcriptome. Dev Cell 2005, 8(4):517-27.

43. Dai X, Zhao PX: psRNATarget: a plant small RNA target analysis server. Nucleic Acids Res 2011, 39(Web Server):W155-9.

44. Kanehisa $M$, et al: KEGG for linking genomes to life and the environment. Nucleic Acids Res 2008, 36(Database):D480-4

45. Barakat $A$, et al: Conservation and divergence of microRNAs in Populus. BMC Genomics 2007, 8:481.

46. Morin RD, et al: Comparative analysis of the small RNA transcriptomes of Pinus contorta and Oryza sativa. Genome Res 2008, 18(4):571-84.

47. Hasegawa PM, et al: Plant Cellular and Molecular Responses to High Salinity. Annu Rev Plant Physiol Plant Mol Biol 2000, 51:463-499.
48. Ding $D$, et al: Differential expression of miRNAs in response to salt stress in maize roots. Ann Bot 2009, 103(1):29-38.

49. Jain $\mathrm{M}$, et al: F-box proteins in rice. Genome-wide analysis, classification, temporal and spatial gene expression during panicle and seed development, and regulation by light and abiotic stress. Plant Physiol 2007, 143(4):1467-83.

50. Jones-Rhoades MW, Bartel DP: Computational identification of plant microRNAs and their targets, including a stress-induced miRNA. Mol Cell 2004, 14(6):787-99.

51. Navarro $L$, et al: A plant miRNA contributes to antibacterial resistance by repressing auxin signaling. Science 2006, 312(5772):436-9.

52. Fedoroff NV: Cross-talk in abscisic acid signaling. Sci STKE 2002, 2002(140), re10.

53. Himmelbach A, Yang Y, Grill E: Relay and control of abscisic acid signaling. Curr Opin Plant Biol 2003, 6(5):470-9.

54. Achard $\mathrm{P}$, et al: Integration of plant responses to environmentally activated phytohormonal signals. Science 2006, 311(5757):91-4.

55. Leyser O: Dynamic integration of auxin transport and signalling. Curr Biol 2006, 16(11):R424-33

56. S P, A K , A P: Identification and validation of conserved microRNAs under salt stress. Plant Cell Tissue Organ Culture 2011, 105:233-242.

57. Ren $Y$, et al: Identification and characterization of salt-responsive microRNAs in Populus tomentosa by high-throughput sequencing. Biochimie 2012, 95(4):743-50.

58. Li WX, et al: The Arabidopsis NFYA5 transcription factor is regulated transcriptionally and posttranscriptionally to promote drought resistance. Plant Cell 2008, 20(8):2238-51.

59. Zhou J, et al: Expression profile of miRNAs in Populus cathayana L. and Salix matsudana Koidz under salt stress. Mol Biol Rep 2012, 39(9):8645-54

60. Ullrich TC, Blaesse M, Huber R: Crystal structure of ATP sulfurylase from Saccharomyces cerevisiae, a key enzyme in sulfate activation. Embo $J$ 2001, 20(3):316-29

61. Liu $\mathrm{Q}$, et al: Expression analysis of phytohormone-regulated microRNAs in rice, implying their regulation roles in plant hormone signaling. FEBS Lett 2009, 583(4):723-8.

62. Gao P, et al: Over-expression of osa-MIR396c decreases salt and alkali stress tolerance. Planta 2010, 231(5):991-1001.

63. Klevebring $D$, et al: Genome-wide profiling of populus small RNAs. BMC Genomics 2009, 10:620.

doi:10.1186/1471-2156-15-S1-S6

Cite this article as: $\mathrm{Si}$ et al:: Genome-wide analysis of salt-responsive and novel microRNAs in Populus euphratica by deep sequencing. BMC Genetics 2014 15(Suppl 1):S6.

\section{Submit your next manuscript to BioMed Central and take full advantage of:}

- Convenient online submission

- Thorough peer review

- No space constraints or color figure charges

- Immediate publication on acceptance

- Inclusion in PubMed, CAS, Scopus and Google Scholar

- Research which is freely available for redistribution 